\title{
Isolamento químico e validação analítica por cromatografia líquida de alta eficiência de quercitrina em Solidago chilensis Meyen (Asteraceae)
}

\author{
VECHIA,C.A.D.'; MORAIS, B.1; SCHONELL, A.P.1; DIEL, K.A.P.1; FAUST, C.1; MENIN,C.1; GOMES, D.B.2; \\ ROMAN, JUNIOR W.A.1,2* \\ 'Universidade Comunitária da Região de Chapecó, Programa de Pós-graduação em Farmacologia Clínica, Rua \\ Sen. Attílio Fontana, 591-E, Efapi, Chapecó, SC, 89809-000. 2Universidade Comunitária da Região de Chapecó, \\ Programa de Pós-graduação em Ciências da Saúde (PPGCS), Rua Sen. Attílio Fontana, 591-E, Efapi, Chapecó, \\ SC, 89809-000. *Autor para correspondência: romanwa@unochapeco.edu.br
}

\begin{abstract}
RESUMO: A espécie Solidago chilensis Meyen, Asteraceae é conhecida como erva-lanceta ou arnica-brasileira, sendo utilizada popularmente como antimicrobiana e para o tratamento de inflamações tópicas. No entanto, estudos fitoquímicos e farmacológicos para as partes aéreas são escassos. Neste trabalho, realizou-se a determinação de flavonoides por espectrofotometria de UV/Vis, prospecção fitoquímica da fração acetato de etila visando o isolamento do constituinte químico majoritário e validação analítica por cromatografia líquida de alta eficiência (CLAE). O teor de flavonoides totais foi de $5,42 \%$, representados como hiperosídeo. $\mathrm{O}$ fracionamento químico utilizando métodos cromatográficos (cromatografia líquida em coluna gel de sílica; $\mathrm{CHCl}_{3}: \mathrm{EtOH} ; 8: 2 \mathrm{v} / \mathrm{v}$ ) e espectroscópicos ( ${ }^{1} \mathrm{H} \mathrm{RMN},{ }^{13} \mathrm{C} \mathrm{RMN} \mathrm{e}$ ESI-MS) revelou o isolamento de quercetina-3-O- $\alpha$-L-ramnosídeo(quercitrina). A sensibilidade e a linearidade $(r=0,999)$ da validação analítica, utilizando a quercitrina isolada do extrato hidroalcoólico da planta, revelaram um rendimento de $5,29 \%$ do analito em relação à droga vegetal. Precisão, recuperação e robustez, além dos valores estabelecidos para os limites de detecção (LOD) e de quantificação (LOQ), poderão ser utilizados como parâmetros de qualidade para extratos à base de $\mathrm{S}$. chilensis.
\end{abstract}

Palavras-chave: Solidago chilensis, quercitrina, validação analítica, CLAE.

\begin{abstract}
Chemical isolation and analytical validation by high-performance liquid chromatography of quercitrin in Solidago chilensis Meyen (Asteraceae). The species Solidago chilensis Meyen Asteraceae, known as "erva-lanceta" or "Brazilian arnica", is popularly used as an antimicrobial and topical treatment for inflammations. However, phytochemical and pharmacological studies of its aerial parts are scarce. In this study, flavonoids were determined by UV/Vis spectrophotometry and phytochemical screening of the ethyl acetate fraction with the goal of isolating the major chemical constituent and analytically validating it through high performance liquid chromatography (HPLC). The total flavonoid content was $5.42 \%$, represented as hyperoside. Chemical fractionation using chromatographic (liquid chromatography in column of silica gel, $\mathrm{CHCl} 3: \mathrm{EtOH}, 8: 2 \mathrm{v} / \mathrm{v}$ ) and spectroscopic methods (1H RMN, 13C RMN, and ESI-MS) revealed the isolation of quercetin-3-O- $\alpha$-L-rhamnoside (quercitrin). The sensitivity and linearity $(r=0.999)$ using the isolated quercitrin of the hydroalcoholic extract of the plant revealed a yield of $5.29 \%$ of analyte in relation to the plant. Precision, recovery, and robustness, as well as values set for the limits of detection (LOD) and quantitation (LOQ) can be used as quality parameters for extracts based on S. chilensis.
\end{abstract}

Keywords: Solidago chilensis, quercitrin, analytical validation, HPLC.

\section{INTRODUÇÃO}

O gênero Solidago abrange cerca de 120 espécies distribuídas por todo o mundo, sendo amplamente utilizadas na medicina tradicional de vários países (Assiniet al., 2013). Na América do Sul, a espécie nativa mais encontrada é Solidago chilensis Meyen (Asteraceae), conhecida 
popularmente como erva-lanceta, espiga-de-ouro ou arnica-brasileira. As partes aéreas da planta são utilizadas em preparados caseiros, como antimicrobiana, analgésica, cicatrizante e antiinflamatória de uso tópico (Goulart et al., 2007; Assini et al., 2013),em substituição à arnica-verdadeira (Arnica Montana L.) (Lorenzi \& Matos, 2002).

Os principais constituintes químicos são os diterpenoides (Valverde et al., 2012), óleos essenciais, saponinas, acetofenona (Silva et al., 2010), $\beta$-farneseno, $\alpha$-amirina, ésteres de ácidos carboxílicos (Smolarek et al., 2009), sendo o flavonoide quercitrina, a substância química majoritária (Torres et al., 1987).

O extrato aquoso das inflorescências de S.chilensis revelaram atividades gastroprotetoras em camundongos utilizando o modelo de indução com ácido acético (Schemeda-Hirschmannet al., 2002), sem apresentar toxicidade nas concentrações de 125 a 2000 mg/kg (Bucciarelli et al.,2010). A atividade anti-inflamatória foi descrita por Tamura et al. (2009) utilizando os métodos de edema de pata e migração de células polimorfonucleares. Foram observados efeitos antimicrobianos (Morel et al., 2006), antioxidantes (Apáti et al., 2006) e de citotoxicidade (Martins et al., 2009). Recentemente, Silva et al. (2010) relataram, em estudo clínico, a atividade anti-inflamatória do extrato hidroalcoólico frente ao lumbago.

No entanto, sabe-se que os extratos vegetais apresentam uma ampla variabilidade e complexidade em sua composição química devido a fatores ambientais e climáticos. Dessa forma, é fundamental a continuidade de estudos sobre os parâmetros de qualidade, bem como, a validação de métodos analíticos (Hostettmann et al., 2003). O desenvolvimento e a validação de um método analítico eficiente é parte integrante para o registro de fitoterápicos e objetiva a garantia de segurança e eficácia do produto resultante (Shabir, 2003; Marques et al., 2012).

$\mathrm{Na}$ atualidade tem crescido a necessidade de demonstrar a qualidade de medições químicas, através de sua comparabilidade, rastreabilidade e confiabilidade dos dados analíticos (Brito et al., 2003; Ribaniet al., 2004); para esta finalidade, esses necessitam passar pelo processo de validação (Alves et al., 2010). No Brasil, a Agência Nacional de Vigilância Sanitária (Anvisa), baseada na International Conference on Harmonisation of Technical Requirements for Registration of Pharmaceuticals for Human Use (ICH, 1996), publicou a Resolução n899/2003, que estabelece parâmetros para validação de métodos bioanalíticos aplicáveis a produtos farmacêuticos (Anvisa, 2003).

Técnicas de separação como a cromatografia líquida de alta eficiência (CLAE) e a cromatografia líquida de coluna (CLC) podem ser empregadas para validação, pela capacidade de identificação de compostos por comparação com padrões e de purificação de substâncias separandoos de componentes de uma mistura, seja ela biológica, seja farmacêutica ou alimentícia (Degani et al., 1998; Ribani et al., 2004). Particularmente, a CLAE é largamente empregada para extratos vegetais por possuir vantagens, como curto tempo de preparo da amostra a ser analisada; dados qualitativos e quantitativos podem ser obtidos para amostras de polaridades variadas em uma só corrida de análise, e permite várias formas de detecção, podendo aumentar a especificidade e o conteúdo de informações por ensaio (Karcher et al., 2005).

Apesar de S. chilensis estar inserida na Relação Nacional de Plantas Medicinais de Interesse ao Sistema Único de Saúde (SUS) (Brasil, 2009), e seu extrato já ter sido avaliado em estudos clínicos (Silva et al., 2010), ainda não foram realizados estudos de validação analítica de seu constituinte químico majoritário.

Este trabalho visou o isolamento químico de quercitrina e o desenvolvimento de uma metodologia analítica para determinação e quantificação desta substância no extrato hidroalcoólico das partes aéreas de $S$. chilensis. O método proposto foi validado de acordo com a resolução da Anvisa e diretrizes da $\mathrm{ICH}$ para determinação da especificidade, linearidade, limite de detecção e quantificação, exatidão, precisão, robustez e reprodutibilidade.

\section{MATERIAIS E MÉTODOS Material vegetal}

As partes aéreas de Solidago chilensis Meyen foram coletadas (03/2014) na cidade de Pinhalzinho (SC), Brasil (S 26 50' 53"; W 52 59' 31"). O material vegetal foi identificado por Osmar dos Santos Ribas, curador do herbário do Museu Botânico Municipal de Curitiba (PR), onde a exsicata está depositada (MBM 356792).

\section{Solventes e reagentes}

Os solventes acetonitrila $\left(\mathrm{CH}_{3} \mathrm{CN}\right)$, ácido acético $(\mathrm{AcOH})$ e metanol $(\mathrm{MeOH})$ utilizados foram grau HPLC (Sigma ${ }^{\circledR}$, USA). A água foi obtida por ultrapurificador de água Milli-Q A10 (Gradiente ${ }^{\circledR}$ ) e os solventes $\mathrm{MeOH}$, acetato de etila (AcOEt), n-hexano, etanol $(\mathrm{EtOH})$ e clorofórmio $\left(\mathrm{CHCl}_{3}\right)$ foram de grau analítico (Tedia ${ }^{\circledR}$, USA).

\section{Preparação do extrato hidroalcoólico de Solidago chilensis (EHS)}

O material vegetal foi submetido à secagem em temperatura ambiente, protegido da luz direta 
e umidade. As partes áreas foram trituradas em moinho de facas e selecionadas em tamis de 425 um (35 Tyler/Mesh). O extrato fluido foi obtido por percolação hidroalcoólica (etanol a $70 \% \mathrm{v} / \mathrm{v}$ ) utilizando material vegetal dessecado de S.chilensis (50 g), conforme preconizado pela FB 5 (2010). O extrato hidroalcoólico (EHS) foi concentrado em rotavapor sob pressão reduzida a uma temperatura de $40^{\circ} \mathrm{C}$, liofilizado e estocado $\mathrm{a}-20^{\circ} \mathrm{C}$.

\section{Determinação de flavonoides totais}

A determinação de flavonoides totais das partes aéreas da planta foi realizada por meio de método farmacopeico (Farmacopeia Brasileira IV, 1988-2005). Brevemente, a droga vegetal $(0,400$ g; $425 \mu \mathrm{m}$ ) foi submetida extração sob refluxo (50 min) utilizando como solvente acetona $(60 \mathrm{~mL})$. $O$ filtrado foi submetido à partição com acetato de etila ( $45 \mathrm{~mL}$ ) e na fase orgânica foi adicionado $(1 \mathrm{~mL})$ de cloreto de alumínio $(2 \%$ em metanol; $\mathrm{p} / \mathrm{v})$. Após $30 \mathrm{~min}$ foram realizadas as análises de espectrofotometria UV/Vis em comprimento de onda de $425 \mathrm{~nm}$ com número de repetições igual a três. A porcentagem de flavonoides totais foi expressa segundo a fórmula:

$$
\mathrm{TFT}=\frac{A b s \cdot 62,500}{500 \cdot m \cdot(100-\mathrm{PD})}(\% ; p / p)
$$

Abs = absorvância;

$\mathrm{m}$ = massa da droga $(\mathrm{g})$;

$\mathrm{PD}=$ perda por dessecação $(\% ; p / p)$

$O$ resultado é fornecido em porcentual $(p / p)$ de flavonoides totais calculados como hiperosídeo $\left(\mathrm{C}_{21} \mathrm{H}_{20} \mathrm{O}_{12}\right)$.

\section{Isolamento químico}

Uma amostra do extrato EHS $(20 \mathrm{~g})$ foi diluída em béquer com água ( $200 \mathrm{~mL}$ ) e submetida à agitação mecânica (20 min). Posteriormente, a solução foi transferida para o funil de separação e efetuaram-se partições $(n=10)$ com solventes de polaridade crescente ( $n$-hexano e AcOEt). Uma amostra da fração AcOEt $(5,0 \mathrm{~g})$ foi dissolvida em quantidade suficiente de $\mathrm{CHCl}_{3}$ e submetida à cromatografia líquida em coluna utilizando como fase estacionária gel de sílica (Merck ${ }^{\circledR}$, Alemanha) e eluente $\mathrm{CHCl}_{3}$ e EtOH (80:20 v/v) em polaridade crescente até EtOH $80 \%(\mathrm{v} / \mathrm{v})$. O fracionamento rendeu 8 subfrações $(5 \mathrm{~mL})$ que foram reunidas por semelhança em cromatografia de camada delgada (CCD) empregando como fase móvel AcOEt:MeOH: $\mathrm{H}_{2} \mathrm{O}(100: 13,5: 10 \mathrm{v} / \mathrm{v})$ e analisadas em câmara de UV/Vis em $366 \mathrm{~nm}$. A subfração 6 foi encaminhada para análises espectroscópicas $\left({ }^{1} \mathrm{H}\right.$ $\mathrm{RMN},{ }^{13} \mathrm{C}$ RMN e EM-ESI).

\section{Método de validação Especificidade e seletividade}

A especificidade e a seletividade do método para determinação de quercitrina no EHS foram realizadas por meio de varreduras entre os comprimentos de onda de 200 e $800 \mathrm{~nm}$ em espectrofotômetro UV/Vis (Biochrom ${ }^{\circledast}$, Libra S22) e por análise em cromatografia líquida de alta eficiência (CLAE). Para análises em CLAE, uma amostra de EHS (50 mg) foi dissolvida em $50 \mathrm{~mL}$ de $\mathrm{MeOH}(1 \mathrm{mg} / \mathrm{mL})$, submetida à ultrassom e filtrada (Micropore ${ }^{\circledast}(0,45 \mathrm{~mm})$ em ultrafiltro Sartorius Stedium, Biotech ${ }^{\circledR}$. A solução padrão foi produzida utilizando a quercitrina isolada de $\mathrm{S}$. chilensis $(30$ $\mathrm{mg})$, dissolvida em MeOH (30 mL) $(1 \mathrm{mg} / \mathrm{mL}) \mathrm{com}$ posterior filtração. As análises foram realizadas utilizando equipamento Varian ${ }^{\circledR}$ Pró-Star com injetor manual (alça de $20 \mu \mathrm{L}$ ), gradiente ternário de bombas, detector UV/Vis e coluna Kromasil@ ODS $(5 \mu \mathrm{m})$ fase reversa C-18 $(25 \times 4,5 \mathrm{~mm}) \mathrm{em}$ temperatura de $24 \pm 2^{\circ} \mathrm{C}$. Dois sistemas de solventes foram utilizados para a análise, $\mathrm{H}_{2} \mathrm{O}$ : $\mathrm{AcOH}(40: 1$ $\mathrm{v} / \mathrm{v}$ ) (solvente $\mathrm{A}$ ) e $\mathrm{CH}_{3} \mathrm{CN}$ (solvente $\mathrm{B}$ ). A análise foi realizada com vazão de $1 \mathrm{~mL} / \mathrm{min}$. O gradiente utilizado constituiu-se de $86 \%$ de A por 15 min, $65 \%$ de A por 30 min e $100 \%$ de B por 2 min. A detecção por UV foi efetuada em 360 nm (Apáti et al., 2003).

\section{Linearidade}

A linearidade para quantificação de quercitrina no EHS foi avaliada por meio de curva analítica utilizando concentrações crescentes de quercitrina $(12,5 ; 25,50,100,200$ e $400 \mu \mathrm{g} / \mathrm{mL})$. As análises cromatográficas em CLAE foram realizadas em triplicata e os dados para área do pico versus concentração da droga foram tratados por análise de regressão linear por meio do método dos mínimos quadrados.

\section{Precisão e precisão intermediária}

A precisão do método foi avaliada levando em consideração a repetibilidade e a precisão intermediária. Para a repetibilidade foram preparadas três concentrações de quercitrina em (100, 200 e $400 \mu \mathrm{g} / \mathrm{mL}$ ) e realizaram-se três determinações para cada amostra $(n=3)$. As análises foram realizadas pelo mesmo operador no mesmo dia. Para avaliação da precisão intermediária, empregou-se a mesma metodologia, utilizando outro operador em três dias alternados. A precisão e a precisão intermediária foram expressas com o coeficiente de variação relativo das concentrações de quercitrina.

\section{Recuperação}

A exatidão foi avaliada em termos de recuperação através do método de adição de padrão a amostra. Este método é usado quando é

Rev. Bras. PI. Med., Campinas, v.18, n.1, supl. I, p.288-296, 2016. 
difícil ou impossível preparar um branco da matriz sem a substância de interesse. Foram preparadas diferentes concentrações (baixa, média e alta) de quercitrina em $\mathrm{MeOH}(25,50,100$ e $200 \mu \mathrm{g} /$ $\mathrm{mL})$. Estas soluções $(\mathrm{n}=3)$ foram adicionadas ao material vegetal e submetidas à extração conforme metodologia de preparo da amostra. A amostra sem adição de padrão (EHS) e cada uma das amostras fortificadas com o padrão foram analisadas e a recuperação foi expressa em termos de porcentagem da quantidade medida da substância em relação à quantidade adicionada na matriz.

Limite de detecção (LOD) e limite de quantificação (LOQ)

A estimativa do limite de detecção (LOD) e do limite de quantificação (LOQ) foram calculados com base na relação de sinal/ruído $(\mathrm{S} / \mathrm{N})$ de diluições sucessivas do padrão quercitrina, levando em consideração três e dez vezes a intensidade do sinal ruído (Barbosa et al., 2014), de acordo com a expressão S/N x 3 para LOD e S/N x 10 para LOQ.

\section{Robustez}

Três soluções de amostras foram preparadas e analisadas nas condições estabelecidas e por alteração na taxa de fluxo $(0,9$ e $1,1 \mathrm{~mL} / \mathrm{min}), \mathrm{pH}$ $(1,95$ e 2,02$)$ e mudança no gradiente inicial $(12 \%$ de B e $20 \%$ de B). A avaliação da robustez deu-se por análise da variação na concentração de quercitrina e/ou tempo de retenção.

\section{Análise estatística}

Os resultados foram representados por meio da média \pm desvio padrão (DP) e coeficiente de variação (\%).

\section{RESULTADO E DISCUSSÃO}

\section{Determinação de flavonoides}

O teor de flavonoides totais obtidos das partes aéreas de $S$. chilensis foi de $5,42 \% \pm 0,11(4,55)$ (média $\pm \mathrm{DP})(\mathrm{CV})$ representados como hiperosídeo.
Apáti et al. (2003) revelaram um rendimento de $6,16 \%$ em flavonoides monoglucosídeos obtidos do extrato hidrometanólico $(50 \% \mathrm{v} / \mathrm{v})$ da parte aérea da planta, confirmando que $S$. chilensis possui grande quantidade destas substâncias em extratos hidroalcoólicos.

Isolamento químico de quercitrinade EHS

O perfil cromatográfico da subfração $6 \mathrm{em}$ CCD revelou bandas cromatográficas com fator de retenção ( $R f)$ semelhante ao observado para o padrão quercitrina (Maciel et al., 2006). As análises espectroscópicas de ${ }^{1} \mathrm{H}$ RMN,${ }^{13} \mathrm{C}$ RMN (Tabela 1), juntamente com dados de EM-ESI sugerem fórmula molecular $\mathrm{C}_{21} \mathrm{H}_{20} \mathrm{O}_{11}(448,3 \mathrm{Da})$, que, comparados com dados da literatura (Correia et al., 2008), permitiram confirmar o isolamento da substância quercetina-3-O- $\alpha$-L-ramnosídeo (quercitrina) (Figura 1).

\section{Validação analítica \\ Seletividade}

A seletividade é definida como a capacidade do método em determinar o analito na presença de outras substâncias presente na amostra (Causon, 1997; Thompson et al., 2002). Para os métodos cromatográficos, a seletividade é avaliada no sentido de garantir que o pico de resposta do analito seja proveniente exclusivamente desse, e não de outros compostos (interferentes) presentes na amostra. Para tanto, é conveniente a utilização de testes depureza de pico com auxílio de detector de arranjo de fotodiodos ou de espectrometria de massas (Paschoal et al., 2008). Para a escolha de um método analítico seletivo, inicialmente, realizaram-se varreduras em espectrometria de UV/ Vis utilizando a solução do branco $(\mathrm{MeOH})$, EHS e quercitrina nos comprimentos de onda de 200 a $750 \mathrm{~nm}$. Foram observadas absorbâncias máximas próximas a 250 e $360 \mathrm{~nm}$ (Figura 2), que, conforme Landin et al. (2013), estão relacionados com as substâncias flavonoídicas.

$\mathrm{Na}$ análise cromatográfica de quercitrina

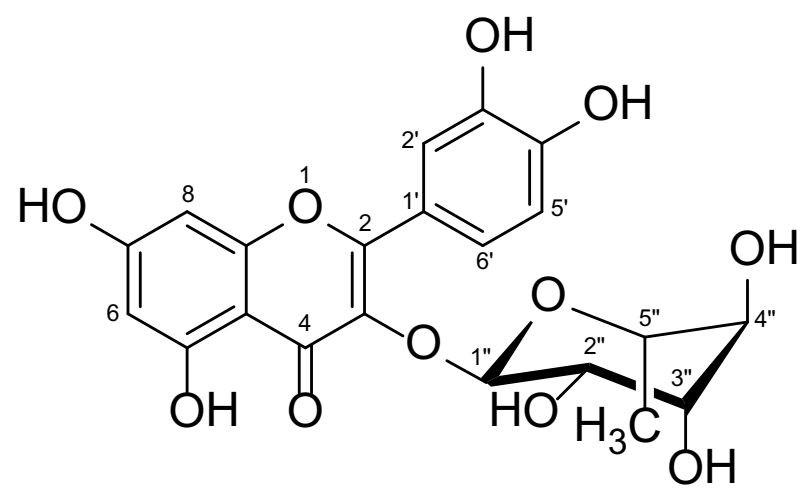

FIGURA 1. Estrutura química da quercetina-3-O- $\alpha$-L-ramnosídeo (quercitrina). 
TABELA 1. Deslocamentos químicos de $\mathrm{RMN}$ de ${ }^{1} \mathrm{H}\left(400 \mathrm{MHz} ; \mathrm{CD}_{3} \mathrm{OD}\right)$ e $\mathrm{RMN}$ de ${ }^{13} \mathrm{C}\left(100 \mathrm{MHz}\right.$; $\left.\mathrm{CD}_{3} \mathrm{OD}\right)$ para quercitrina.

\begin{tabular}{|c|c|c|c|}
\hline $\begin{array}{l}\text { Posições dos } \\
\text { hidrogênios }\end{array}$ & $\begin{array}{c}\text { Deslocamentos de RMN de } 1 \mathrm{H} \\
(\delta=\mathrm{ppm}) \text { (acoplamentos spin-spin) }\end{array}$ & $\begin{array}{c}\text { Deslocamentos de } \\
\text { RMN de } 13 \mathrm{C}(\delta=\mathrm{ppm})\end{array}$ & $\begin{array}{l}\text { Deslocamentos de RMN de } 13 \mathrm{C}(\delta= \\
\text { ppm em } \mathrm{CDCl} 3) \text { Correia et al. (2008) }\end{array}$ \\
\hline 1 & - & - & - \\
\hline 2 & - & 159,4 & 158,4 \\
\hline 3 & - & 136,4 & 136,1 \\
\hline 4 & - & 179,8 & 179,5 \\
\hline 5 & - & 163,4 & 163,0 \\
\hline 6 & $6,20(\mathrm{~d} ; J=2,1 \mathrm{~Hz} ; 1-\mathrm{H})$ & 100,0 & 100,0 \\
\hline 7 & - & 166,0 & 166,3 \\
\hline 8 & $6,37(\mathrm{~d} ; J=2,1 \mathrm{~Hz} ; 1-\mathrm{H})$ & 94,9 & 94,9 \\
\hline 9 & - & 158,7 & 158,2 \\
\hline 10 & - & 106,0 & 105,7 \\
\hline $1^{\prime}$ & - & 123,1 & 122,9 \\
\hline $2^{\prime}$ & $7,34(\mathrm{~d} ; J=2,1 \mathrm{~Hz} ; 1-\mathrm{H})$ & 117,1 & 116,4 \\
\hline $3^{\prime}$ & - & 146,5 & 146,4 \\
\hline $4^{\prime}$ & - & 150,0 & 149,8 \\
\hline $5^{\prime}$ & $6,91(\mathrm{~d} ; J=8,3 \mathrm{~Hz} ; 1-\mathrm{H})$ & 116,5 & 116,9 \\
\hline 6 & $7,31(\mathrm{dd} ; J=8,3: 2,1 \mathrm{~Hz} ; 1-\mathrm{H})$ & 123,0 & 122,9 \\
\hline $1 "$ & $5,35(\mathrm{~d} ; J=1,5 \mathrm{~Hz} ; 1-\mathrm{H})$ & 103,7 & 103,5 \\
\hline $2 "$ & $4,23(\mathrm{dd} ; J=3,4: 1,5 \mathrm{~Hz} ; 1-\mathrm{H})$ & 72,0 & 71,9 \\
\hline $3 "$ & $4,76(\mathrm{dd} ; J=9,4: 3,4 \mathrm{~Hz} ; 1-\mathrm{H})$ & 72,2 & 72,1 \\
\hline $4 "$ & $3,35(\mathrm{dd} ; J=9,4: 3,4 \mathrm{~Hz} ; 1-\mathrm{H})$ & 72,2 & 73,2 \\
\hline $5 "$ & $3,43(\mathrm{dq} ; J=9,4: 6,1 \mathrm{~Hz} ; 1-\mathrm{H})$ & 73,4 & 71,9 \\
\hline $6 "$ & $0,94(\mathrm{~d} ; J=6,1 \mathrm{~Hz} ; 3-\mathrm{H})$ & 17,8 & 17,6 \\
\hline
\end{tabular}

Nota: Em parênteses, d: dupleto; dd: duplo dupleto; dq: duplo quarteto e constante de acoplamento, $\mathrm{J}$ em $\mathrm{Hz}$.

isolada de $S$. chilensis, observa-se uma banda com tempo de retenção de 9,82 min. O cromatograma de EHS revela semelhança com a quercitrina, evidenciando que esta substância é o constituinte majoritário da planta, conforme Torres et al. (1987) (Figura 3). A Farmacopeia Americana (USP, 1990) ressalta que a seletividade de um método analítico está relacionada com sua habilidade de medir, de forma acurada, um analito na presença de interferências as quais se espera que estejam presentes na matriz da amostra. Com a realização de curva analítica, obteve-se a equação da reta ( $y=4,661 x-26,98)$ e o coeficiente de correlação linear $(0,999)$, sendo possível a determinação da concentração de quercitrina $(5,29 \%)$ nas partes aéreas do vegetal. Resultados muito próximos aos apontados neste trabalho foram obtidos recentemente por Roman Junior et al. (2015), que descreveram o rendimento de $6,5 \%$ deste constituinte químico no extrato hidroalcoólico $(70 \%)$ das partes aéreas da planta produzido por percolação. Na quantificação desta substância emSolidago canadensis L. (Asteraceae), Apáti et al. (2003) relataram um rendimento de 3,39 e $5,75 \%$ de quercitrina na tintura (etanol $70 \%$ ) e extração por Soxhlet, respectivamente, confirmando que os solventes alcoólicos ou hidroalcoólicos extraem maior quantidade deste flavonoide quando comparados a preparações aquosas.

\section{Linearidade}

A linearidade é a resposta obtida em função da concentração do analito, a qual deve ser estudada em um intervalo de concentração apropriado (Lanças, 2004). Este parâmetro de validação permite avaliar a aceitação do resultado do sistema cromatográfico com diferentes concentrações da substância estudada (Ribani et al., 
A

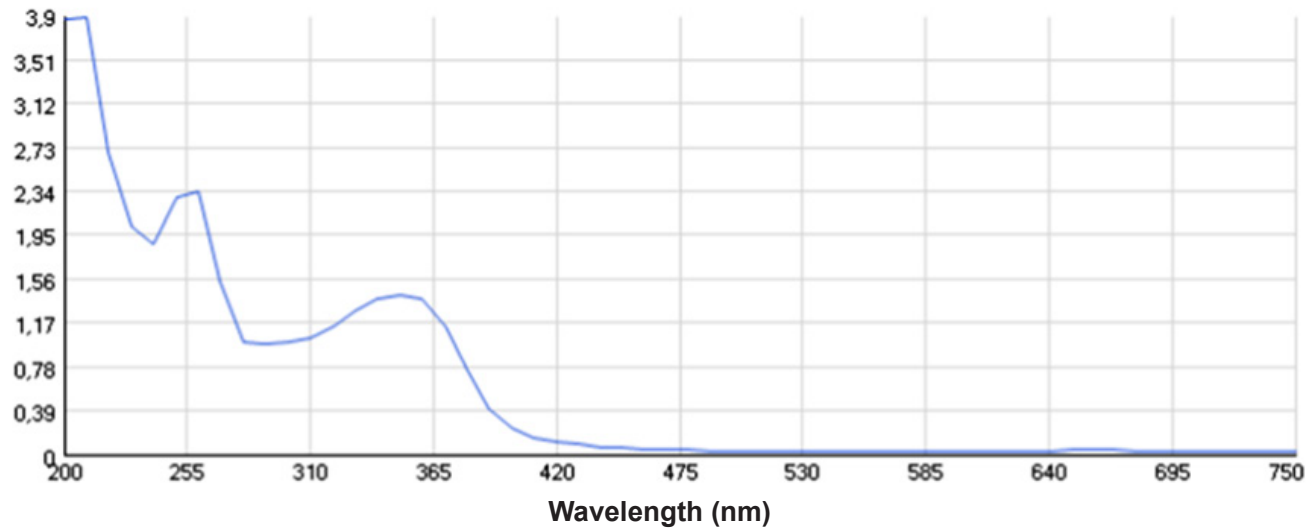

B

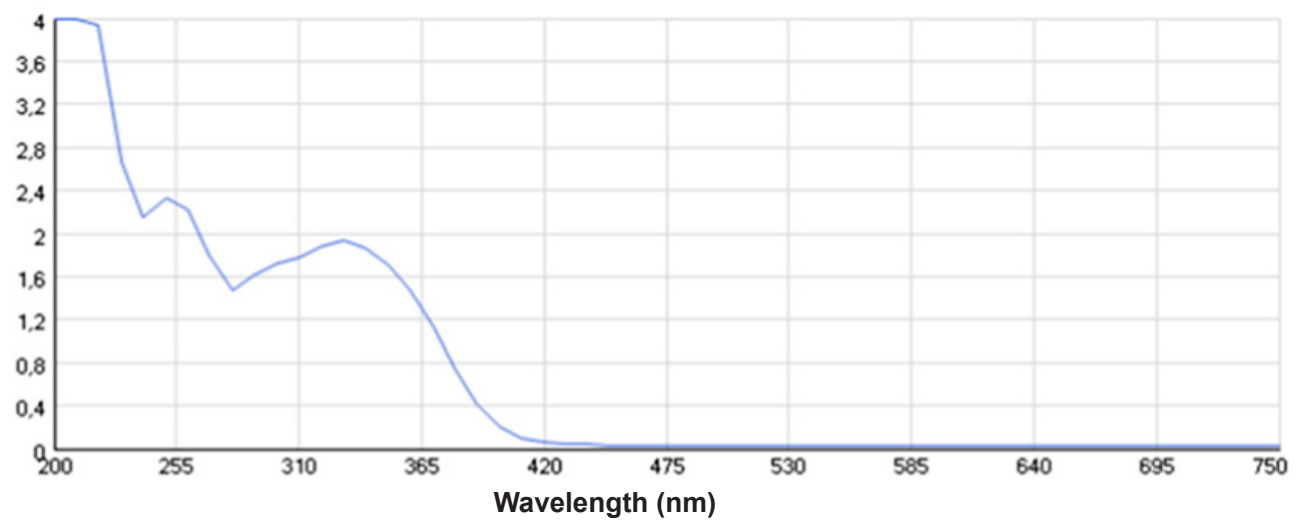

FIGURA 2. Especificidade e seletividadede quercitrina (A) e extrato hidroalcoólico de Solidago chilensis (EHS) (B) por meio de análise em espectofotômetro de UV/Vis (200 a 750 nm).

2004). A linearidade para quercitrina foi realizada utilizando cinco níveis de concentração $(n=3)$, apresentando tempos de retenção em 9,82 min. Obteve-se a equação da reta com y $=4,661 \mathrm{x}-26,98$ e coeficiente de correlação linear 0,999 (Figura 4). Um coeficiente de correlação maior que 0,999 é considerado como evidência de um ajuste ideal dos dados para a linha de regressão. A Anvisa (2003) recomenda um coeficiente de correlação maior ou igual a 0,99 e o Inmetro (2003) considera um valor de $r$ superior a 0,90 como sendo o usualmente requerido.

\section{Precisão e precisão intermediária}

A concordância entre diversos resultados analíticos proporcionados por uma mesma amostra é definida como precisão (Lanças, 2004; Miranda et al., 2015). Neste trabalho,esta expressão foi realizada por meio da repetibilidade das análises em diferentes dias, utilizando diferentes operadores,representadas pelos coeficientes de variação. Os valores obtidos (Tabela 2) apresentam um coeficiente de variação de acordo com o recomendado pela resolução 899/2003 (Anvisa, 2003), que indica como limite um coeficiente de variação de até $5 \%$.

\section{Recuperação}

Os resultados obtidos a partir da análise de exatidão expressam a aceitação entre o valor encontrado e o valor estipulado como nominal ou referência (Lanças, 2004). Os dados revelaram que o método empregado foi capaz de recuperar a quercitrina entre 93 a $108 \%$, apresentando um erro relativo menor que $15 \%$. Este resultado está abaixo dos limites máximos aceitáveis, que são de até $20 \%$ (Ribani et al., 2004).

\section{LOD e LOQ}

O LOD corresponde a menor quantidade que pode ser detectada de um analito em uma amostra, porém, não quantificada com valor exato. $L O Q$ é definido como a menor quantidade quantificada com exatidão de uma substância. As análises revelaram LOD de 1,68 $\mu \mathrm{g} / \mathrm{mL}$ e LOQ de 5 $\mu \mathrm{g} / \mathrm{mL}$ para quercitrina.

\section{Robustez}

A robustez de um método mede a sensibilidade que este apresenta frente a pequenas variações. Diz-se que um método é robusto quando ele não é afetado por uma modificação leve e deliberada em seus parâmetros (Inmetro, 2003), quando esse está sendo executado (Paschoal 

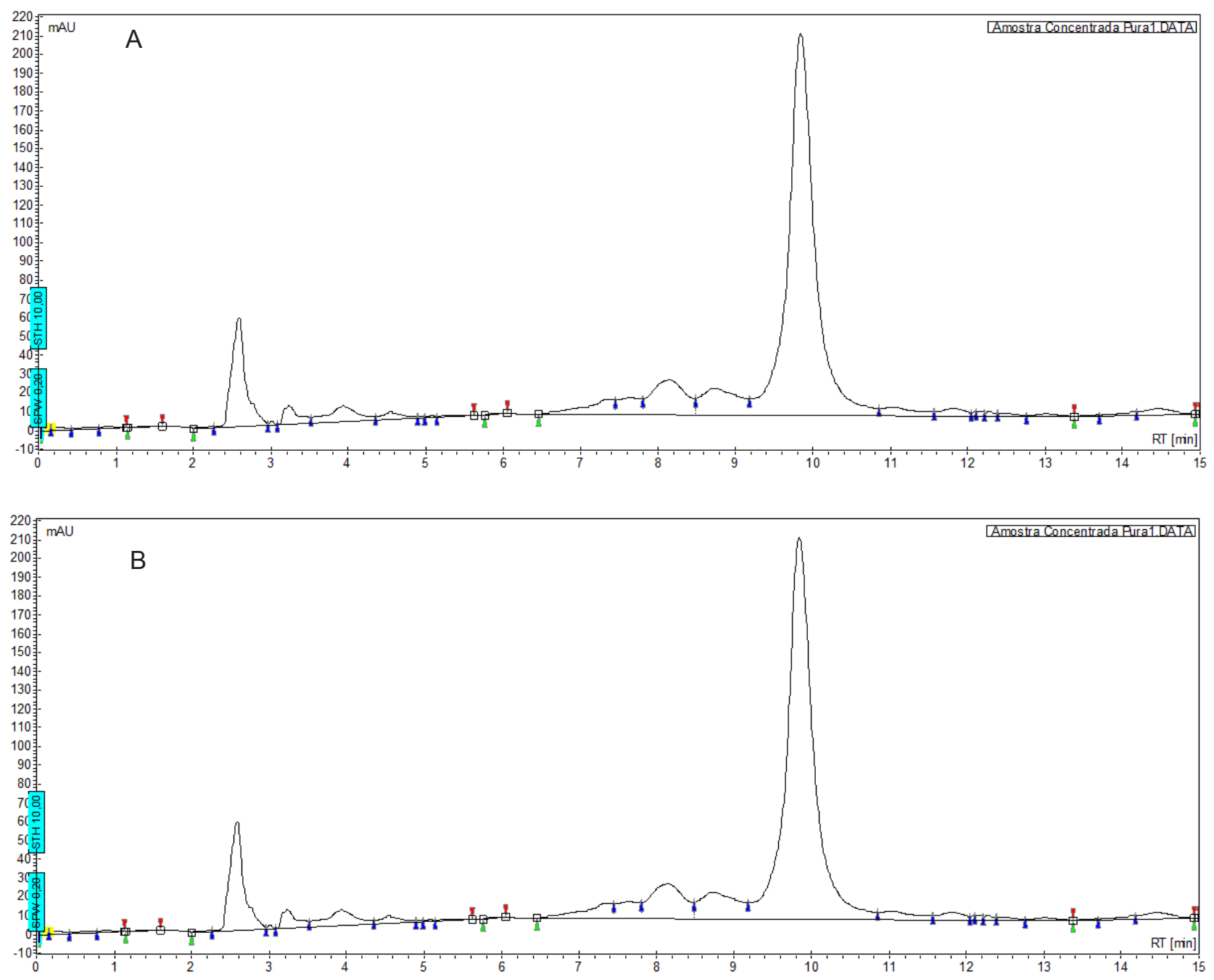

FIGURA 3. Cromatografia líquida de alta eficiência de quercitrina (A) (Rf: $9,82 \mathrm{~min}$ ) e do extrato hidroalcoólico de Solidago chilensis Meyen (Asteraceae) (B). CromatógrafoVarian ${ }^{\oplus}$, coluna Kromasil ${ }^{\circledR}$ ODS $(5 \mu \mathrm{m})$ fase reversa $\mathrm{C}-18(25 \times 4,5 \mathrm{~mm})$ em temperatura de $24 \pm 2^{\circ} \mathrm{C}$. Solventes utilizados: $\mathrm{MeOH}: \mathrm{H}_{3} \mathrm{PO}_{4}(0,1 \% \mathrm{v} / \mathrm{v})$ modo isocrático por $10 \mathrm{~min}$ com vazão de $1 \mathrm{~mL} / \mathrm{min}$ e detecção em $300 \mathrm{~nm}(\mathrm{n}=3)$. $\mathrm{H}_{2} \mathrm{O}: \mathrm{AcOH}(40: 1 \mathrm{v} / \mathrm{v})($ solvente $\mathrm{A})$ e $\mathrm{CH}_{3} \mathrm{CN}$ (solvente B). A análise foi realizada com vazão de $1 \mathrm{~mL} / \mathrm{min}$. O gradiente utilizado constituiu-se de $86 \%$ de $\mathrm{A}$ por $15 \mathrm{~min}, 65 \%$ de A por $30 \mathrm{~min}$ e $100 \%$ de B por $2 \mathrm{~min}$. A detecção por UV foi efetuada em $360 \mathrm{~nm}$.

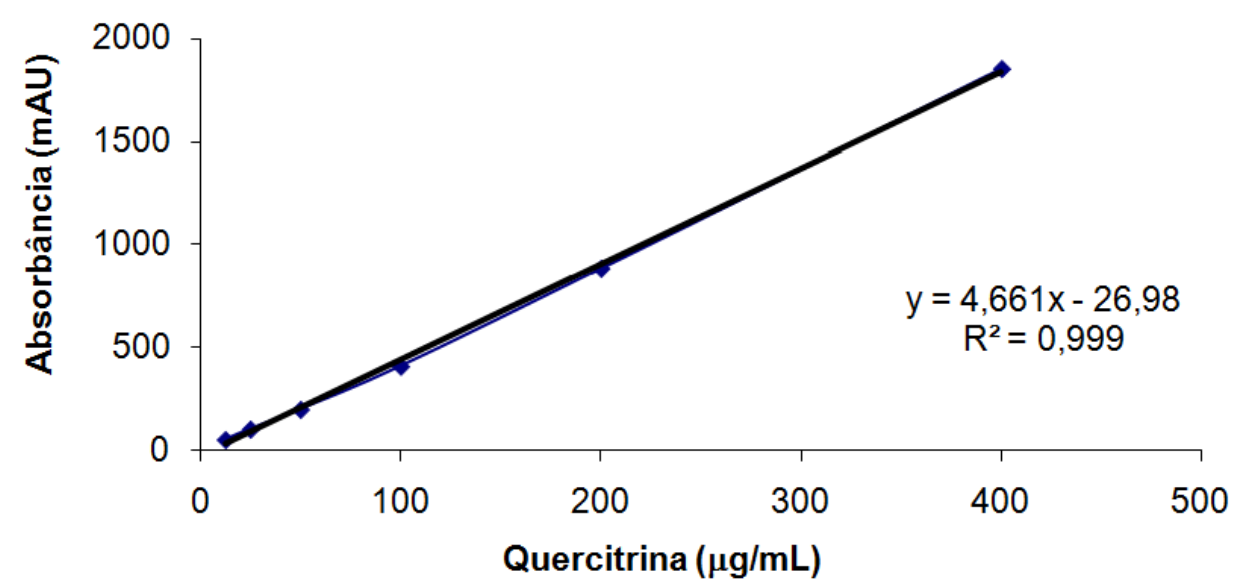

FIGURA 4. Linearidade de quercitrina realizada por meio de curva analítica em cromatografia líquida de alta eficiência (CLAE) $(12,5$ a $400 \mu \mathrm{g} / \mathrm{mL} ; \mathrm{n}=3)$. 
TABELA 2. Coeficientes de variação (\%) para diferentes concentrações de quercitrina nas avaliações de precisão e precisão intermediária $(n=3)$.

\begin{tabular}{lcc}
\hline Teste de validação & Concentração de quercitrina $(\boldsymbol{\mu g} / \mathbf{m L})$ & Coeficiente de variação $(\%)$ \\
\hline \multirow{3}{*}{ Precisão } & 100 & 3,93 \\
& 200 & 2,83 \\
\hline \multirow{2}{*}{ Precisão intermediária } & 400 & 2,59 \\
& 100 & 2,60 \\
& 200 & 4,91 \\
\hline
\end{tabular}

et al., 2008). As análises de robustez em CLAE $(\mathrm{pH}$, gradiente da fase móvel e taxa de fluxo) não apresentaram variações significativas na concentração de quercitrina. O teste apresentou variação significativa no tempo de retenção do analito, quando modificado o gradiente da fase móvel, em que proporcionou um coeficiente de variação de $33 \%$.

\section{CONCLUSÃO}

As partes aéreas da espécie Solidago chilensis apresentam um elevado teor de flavonoides, dentre estes, se destaca a quercitrina como substância majoritária, sendo este flavonoide isolado por meio de métodos cromatográficos e espectroscópicos. O método de validação analítica para quercitrina presente no extrato hidroalcoólico de S. chilensis apresentou seletividade, linearidade, precisão, recuperação e robustez, além de valores estabelecidos de LOD e LOQ conforme preconizados pela Anvisa e ICH. Os resultados obtidos contribuem para a análise fitoquímica, bem como, para parâmetros de qualidade de extratos à base de $\mathrm{S}$. chilensis.

\section{REFERÊNCIAS}

ALVES, L.D.S. et al. Desenvolvimento de Método analítico para quantificação efavirenz por espectrofotometria no UV-VIS. Química Nova, v.33, n.9, p.1967-1972, 2010.

ANVISA - Agência Nacional de Vigilância Sanitária. RE 899, de 29 de maio de 2003. Guia para validação de métodos analíticos e bioanalíticos. Diário Oficial da União, Brasília, DF, 29 de maio de 2003, p. 1-15.

APÁTI, P. et al. Herbal remedies of Solidago-correlation of phytochemical characteristics and antioxidative properties. Journal of Pharmaceutical and Biomedical Analysis, v.32, n.4-5, p.1045-1053, 2003.

APÁTI, P. et al. In-vitro effect of flavonoids from Solidago canadensis extract on glutathione S-transferase. Journal of Pharmacy and Pharmacology, v.58, n.2, p.251-256, 2006.

ASSINI, F.L. et al. Efeito Farmacológico do extrato aquoso de Solidago chilensis Meyen em camundongos. Revista Brasileira Plantas Medicinais, v.15, n.1, p.130-134, 2013.

BARBOSA, E.S. et al. Otimização e validação da extração sólida-líquida e purificação em baixa temperatura de HPAs em lodo de esgoto. Química Nova, v.3, n.3, p.404-410, 2014.

BRASIL. Ministério da Saúde. Relação nacional de plantas medicinais de interesse ao SUS. Brasília: Ministério da Saúde, 2009. Disponível em: <http:// bvsms.saude.gov.br/bvs/publicacoes/programa nacional_plantas_medicinais_fitoterapicos.pdf $>$. Acesso em: 03 jul. 2015.

BRITO, N.M. et al. Validação de métodos analíticos: estratégia e discussão. Pesticidas: Revista Ecotoxicologia e Meio Ambiente, v.13, p.130-146, 2003.

BUCCIARELLI, A. et al. Evaluation of gastroprotective activity and acute toxicity of Solidago chilensis Meyen (Asteraceae). Pharmaceutical Biology, v.48, n.9, p.1025-1030, 2010.

CAUSON, R. Validation of chromatographic methods in biomedical analysis viewpoint and discussion. Journal of Chromatography B, v.689, p.175-180, 1997.

CORREIA, S.J. et al. Flavonoides, noresoprenoides e outros terpenos das folhas de Tapirira guianensis. Química Nova, v.31, n.8, p.2056-2059, 2008.

DEGANI, A.L.G. et al. Cromatografia um breve ensaio. Química Nova na Escola, n.7, p.21-25, 1998.

FARMACOPEIA BRASILEIRA. 4. ed. v.1. Brasília: Agência Nacional de Vigilância Sanitária, 2005. 545p.

FARMACOPEIA BRASILEIRA. 5. ed. v.1 e 2. Brasília: Agência Nacional de Vigilância Sanitária, 2010. 808p.

GOULART, S. et al. Anti-inflammatory evaluation of Solidago chilensis Meyen in a murine model of pleurisy. Journal of Ethnopharmacology, v.113. n.2, p.346353, 2007.

HOSTETTMANN, H.K.; QUEIROZ, E.F.; VIEIRA, P.C. Princípios ativos de plantas superiores. 2.ed. São Carlos: UFSCar, 2003. 234p.

$\mathrm{ICH}$. International Conference on Harmonisation of Technical Requirements for Registration of Pharmaceuticals for Human Use. Validation of analytical procedures: text and methodology Q2 (R1), 1996. Disponível em: http://www.ema.europa.eu/docs/ en_GB/document_library/Scientific_guideline/2009/09/ WC̄500002662.pdf. Acesso em: 13 jul. 2015.

INMETRO. Instituto Nacional de Metrologia, Normalização e Qualidade Industrial. Orientações sobre Validação de Métodos de Ensaios Químicos, DOQ-CGCRE-008, 2003. Disponível em: http://www.inmetro.gov.br/Sidoq/ Arquivos/CGCRE/DOQ/DOQ-CGCRE-8_02.pdf. Acesso em 22 jul. 2015.

KARCHER, B.D. et al. A 21st century HPLC workflow for process. Journal of Laboratory Automation, v.10, p.381-393, 2005.

Rev. Bras. PI. Med., Campinas, v.18, n.1, supl. I, p.288-296, 2016. 
LANÇAS, F.M. Validação de métodos cromatográficos de análise. 1.ed. São Carlos: RiMa, 2004. 62p.

LANDIN, L.P.et al. Development and validation of a HPLC method for the quantification of three flavonoids in a crude extract of Dimorphandra gardneriana. Brazilian Journal of Pharmacognosy, v.23, n.1, p.58-64, 2013.

LORENZI, H.; MATOS, F. Plantas medicinais do Brasil: nativas e exóticas. 1.ed. São Paulo: Instituto Plantarum de Estudos da Flora, 2002. 450p.

MACIEL, R.L. et al. Características físico-químicas e químicas e estudo preliminar de estabilidade de tinturas preparadas com espécies de arnica Lychnophora em comparação com Arnica montana. Brazilian Journal of Pharmacognosy, v.16, n.1, p.99-104, 2006.

MARQUES, G.S. et al. Avaliação de procedimentos para quantificação espectrofotométrica de flavonoides em folhas Bauhinia forficata Link. Química Nova, v.35, n.3, p.517-522, 2012.

MARTINS, M.D. et al. Citoxicidade in vitro do extrato de arnica-brasileira (Solidago microglossa) e arnicapaulista (Porophyllium ruderali). ConScientiae Saúde, v.8, n.1, p.99-104, 2009.

MIRANDA, L. et al. Validação intralaboratorial da determinação de metiltestosterona em águas naturais por voltametria usando eletrodo de gota pendente de mercúrio. Química Nova, v.38, n.3, p.419-426, 2015.

MOREL, A.F. et al. Antimicrobial activity of extractives of Solidago microglossa. Fitoterapia, v.77, p.453455, 2006.

PASCHOAL, J.A.R. et al. Validação de métodos cromatográficos para a determinação de resíduos de medicamentos veterinários em alimentos.Química Nova, v.31, n.5, p.1190-1198, 2008.

RIBANI, M. et al. Validação em métodos cromatográficos e eletroforéticos. Química Nova, v.27, n.5, p.771780, 2004.

ROMAN JUNIOR, et al. Hypolipidemic effects of Solidago chilensis hydroalcoholic extract and its major isolated constituent quercetrin in cholesterol- fed rats. Pharmaceutical Biology, v.53, n.10, p.1488-1495. 2015.

SCHEMEDA-HIRSCHMANN, G. et al. Gastroprotective activity of the diterpene solidagenone and its derivates on experimentally induced gastric lesions in mice. Journal of Ethnopharmacology, v.81, p.111-115, 2002.

SHABIR, G.A.J. Validation of high-performance liquid chromatography methods for pharmaceutical analysis. Understanding the differences and similarities between validation requirements of the US Food and Drug Administration, the US Pharmacopeia and the International Conference on Harmonization. Journal of Chromatography, v.98, p.57-66, 2003.

SILVA, A.G. et al. Avaliation of an extract of Brazilian arnica (Solidago chilensis Meyen, Asteraceae) in treating lumbago. Phytotherapy Research, v.24, p.283-287, 2010.

SMOLAREK, F.S.F. et al. Abordagem fitoquímica e das atividades biológicas da espécie vegetal Solidago microglossa D.C. Visão acadêmica, v.10, n.1, p.7782, 2009.

TAMURA, E.K. et al. Inhibitory effects of Solidago chilensis Meyen hydroalcoholic extract on acute inflammation. Journal of Ethnopharmacology, v.122, n.3, p.478-485, 2009.

THOMPSON, M. et al. Harmonized guidelines for single-laboratory validation of methods of analysis (IUPAC Technical Report). Pure and Applied Chemistry, v.74, n.5, p.835-875, 2002.

TORRES, L.M.B. et al. Quercitrina em Solidago microglossa DC, a arnica do Brasil. Revista de Farmácia e Bioquímica - USP, v.23, n.1, p.33-40, 1987.

USP. United States Pharmacopeia. The US Pharmacopeia Convention Inc XVII. National Formulary. 22.ed. Easton, PA: Mack Printing Coy, 1990, 1712p.

VALVERDE, S.S. et al. Solidago chilensis Meyen (Asteraceae). Revista Fitos, v.7, n.3, p.131-136, 2012.

Rev. Bras. PI. Med., Campinas, v.18, n.1, supl. I, p.288-296, 2016. 